\title{
The effect of coenzyme Q10 on cognitive status in chronic hemodialysis patients; a double-blind, randomized, clinical trial
}

\author{
Mohammad Reza Tamadon $^{1+(0)}$, Majid Mirmohammadkhani ${ }^{2}$, Shahb Rafaati ${ }^{3}$ \\ ${ }^{1}$ Department of Nephrology, Semnan University of Medical Sciences, Semnan, Iran \\ ${ }^{2}$ Social Determinants of Health Research Center, Semnan University of Medical Sciences, Semnan, Iran \\ ${ }^{3}$ Resident of Internal Medicine, Department of Internal Medicine, Semnan University of Medical Sciences, Semnan, Iran
}

\section{A R T I C L E I N F O}

\section{Article Type:}

Original

\section{Article History:}

Received: 12 May 2018

Accepted: 3 July 2018

ePublished: 28 July 2018

\section{Keywords:}

Coenzyme Q10

Hemodialysis

Psychological disorders

Mini-mental state examination

End-stage renal disease

Chronic kidney disease

Glomerular filtration rate

\begin{abstract}
A B S T RA C T
Introduction: Renal failure is a stressful process that has several complications, including neurological disorders such as cognitive disorder, which can lead to the incidence of psychological disorders.

Objectives: Given the reduced coenzyme Q10 levels in patients with chronic renal failure and the known role of this coenzyme in cognitive disorders, the present study was conducted to assess the effect of CoQ10 supplementation in the treatment of cognitive disorder in hemodialysis patients in Semnan, Iran.

Patients and Methods: The present double-blind, randomized, clinical trial was conducted on patients with chronic renal failure undergoing hemodialysis at Kosar Hospital of Semnan. The participants underwent a cognitive assessment using the Mini-Mental State Examination (MMSE). They were then randomly divided into two groups; group one received CoQ10 supplement and group two received placebo for 90 days, after which period they underwent another cognitive assessment using the MMSE. The Kolmogorov-Smirnov test and Levene's test were used to verify the normal distribution of the data. Data were analyzed using the $t$ test and Mann-Whitney $\mathrm{U}$ test at a significance level of $P<0.05$.

Results: There were no statistically significant differences between the two groups in terms of their personal details. The mean ( \pm standard deviation) of the MMSE1 score was $21.29 \pm 6.5$ in the control group and $21.09 \pm 5.4$ in the case group. The mean ( \pm standard deviation) of the MMSE2 score was $20.62 \pm 6.5$ in the control group and $23.29 \pm 5.8$ in the case group. A significant difference between the two groups in terms of their pre- and post-intervention MMSE scores was observed, suggesting the positive effect of the medication $(P<0.001)$.

Conclusion: The daily intake of CoQ10 100-mg led to a significant change in the cognitive status of chronic hemodialysis patients compared to the group that received only the placebo. Conducting a similar study but with the addition of a healthy control group is recommended in order to establish a base measure for the healthy population and to thus enable the better interpretation of the effect of this supplement.
\end{abstract}

Implication for health policy/practice/research/medical education:

Renal failure is a stressful process that has several complications, including neurological disorders such as cognitive disorder, which can lead to the incidence of psychological disorders. Given the reduced coenzyme Q10 (CoQ10) levels in patients with chronic renal failure and the known role of this coenzyme in cognitive disorders, the present study indicated that daily intake of CoQ10 100-mg led to a significant change in the cognitive status of chronic hemodialysis patients compared to the group that received only the placebo. Please cite this paper as: Tamadon MR, Mirmohammadkhani M, Rafaati S. The effect of coenzyme Q10 on cognitive status in chronic hemodialysis patients; a double-blind, randomized, clinical trial. J Nephropharmacol. 2018;7(2):149-155.

\section{Introduction}

Chronic kidney disease (CKD) refers to reduced glomerular filtration rate (GFR) or increased urinary albumin excretion or both and is rising as a public health issue (1). The global prevalence of this disease is estimated at $8 \%$ to $16 \%(2)$. Traditionally, CKD is classified into five stages depending on its severity. Stages one to three are considered the early stages of the disease and are usually asymptomatic (3). Stages four and five are symptomatic, and patients in stage five, called end-stage renal disease (ESRD), need kidney replacement (4). According to a report by the Center for Disease Prevention and Control, one out of every ten Americans is affected by CKD to some extent, and 22.4 million cases $(11.1 \%$ of the US 
population) are in stages one to three of the disease and more than 661 thousand have ESRD, 468000 of which undergo hemodialysis and 193000 have received a kidney transplant $(3,5,6)$.

CKD patients experience many complications, including cognitive disorder as one of the most common (7-10). The incidence of cognitive disorder has a direct relationship with the severity of CKD and the rate of decline in kidney function, so that the risk of cognitive disorder increases in CKD patients with an eGFR $<60 \mathrm{~mL} / \mathrm{min} / 1.73 \mathrm{~m}^{2}$, and for every $10 \mathrm{~mL} / \mathrm{min}$ reduction in GFR, the risk of cognitive disorder increases by $15 \%$ to $25 \%$ (11-15). The prevalence of cognitive disorder in ESRD patients is three times higher than that in the general population and close to $80 \%$ of ESRD patients experience some degree of cognitive disorder (16-18). The pathogenesis of cognitive disorder in chronic hemodialysis patients is essentially unknown (17). It is believed, however, that immunological processes and lipoprotein peroxidation occur in the body with uremia that cause degenerative neurological damage (19-23). Atherosclerosis, another common disease among hemodialysis patients, facilitates the process of nerve damage $(24,25)$. Many studies have therefore examined the protective role of fat-soluble antioxidants against degenerative neurological damage. Coenzyme Q10 is one of these antioxidants $(26,27)$. Plasma concentrations of this antioxidant decline in patients with CKD and chronic hemodialysis patients $(28,29)$. Coenzyme Q10 or ubiquinone is an electron transmitter in the mitochondrial respiratory chain and its dietary supplement (CoQ10) is used extensively in the treatment of mitochondrial and neurodegenerative disorders $(30,31)$.

\section{Objectives}

Considering that cognitive disorder is a common complication in chronic hemodialysis patients, and given the proof on declined CoQ10 levels in patients with chronic renal failure and the role of this coenzyme in degenerative diseases and cognitive disorder (32-34), the present study was conducted to determine the supplemental dietary role of this coenzyme in the treatment of cognitive disorder in patients with CRF undergoing chronic hemodialysis.

\section{Patients and Methods}

\section{Study population and research design}

The present randomized double-blind placebo-controlled trial was conducted on patients undergoing chronic hemodialysis at Kosar hospital in Semnan, Iran, from July to November 2015. The study subjects were selected from all the eligible hemodialysis patients presenting to the select hospital. The subjects were then randomly divided into a case and a control group. The study was approved by the ethics committee of Semnan University of Medical Sciences and informed consent was obtained from all the participating patients. In the case group, the patients were asked to take $100 \mathrm{mg}$ daily doses of CoQ10 at the same hour each day after their hemodialysis session in conjunction with their other medications. The control group received placebos under the same conditions.

The patients underwent cognitive assessment using the Mini-Mental State Examination (MMSE); the validity and reliability of the Persian version of this test have been confirmed in previous studies (35). The MMSE is a brief quantitative test for assessing cognitive status in adults that was developed by Marshal Folstein in 1975 to screen for cognitive disorder, measure the severity of the disorder, follow-up on its progress and examine its response to treatment in patients. This questionnaire consists of 20 items that are given a total score of 30 , and scoring less than 25 is indicative of cognitive disorder. Patients are given 15 minutes to complete this questionnaire (35).

Before conducting the intervention, the patients' cognitive status was assessed using the MMSE; after 90 days, both groups took the MMSE once again and the pre- test and post-test results were compared between the groups and analyzed. Demographic data were collected by surveying the patients and reviewing their medical records.

\section{Ethical issues}

The research followed the tenets of the Declaration of Helsinki. Informed consents were obtained from all patients. The study was approved by the research committee of Semnan University of Medical Sciences (\#695). Besides that, the study protocol was registered in the Iranian Registry of Clinical Trials (identifier: IRCT2014103019758N1; https://en.irct.ir/trial/17594).

\section{Statistical analysis}

Data were analyzed in SPSS-20 using the KolmogorovSmirnov test and Levene's test to verify their normal distribution, using the paired and student t-tests in the case of normally distributed data, and using the Wilcoxon test and Mann-Whitney U test in the case of non-normally distributed data. The level of statistical significance was set at $P<0.05$.

\section{Results}

A total of 80 patients were randomly divided into two equal groups of 40. Six patients from the control group (one died, three withdrew, one had a transplant and one moved to another city) and six others from the case group (one died, two withdrew, two had transplants and one moved to another city) were excluded from the study. Exclusions from both groups were rare and occurred for similar reasons.

The mean age ( \pm standard deviation) was $63.08 \pm 13.9$ years in the control group and $59.55 \pm 14.9$ in the case group, which indicates the lack of significant differences between the two groups in terms of age $(P=0.488)$. The youngest and oldest patients were 30 and 86 years old in the control group and 27 and 84 in the case group (Table 1). 
In the control group, 11 had low literacy, 12 had primary school education, two had junior high school education, six had a high school diploma and one had above high school education. In the case group, ten had low literacy, nine had primary school education, six had junior high school education, eight had a high school diploma and one had above high school education. The two groups were not significantly different in terms of their level of education $(P=0.606)$.

The mean duration of the disease ( \pm standard deviation) was $41.7 \pm 36.5$ months in the control group, $45.55 \pm 30.8$ months in the case group and $43.62 \pm 33.6$ months in total. After eliminating the excluded patients, this rate changed to $42.32 \pm 38.9$ months in the control group, $48.44 \pm 32.6$ months in the case group and $45.38 \pm 35.8$ months in total. There were no significant differences between the two groups regarding their duration of disease $(P=0.612)$. Table 2 presents the mean, standard deviation, maximum and minimum duration of disease in the two groups.

The mean MMSE1 score ( \pm standard deviation) was $21.29 \pm 6.5$ in the control group and 21.09 \pm 5.4 in the case group. The mean MMSE2 score ( \pm standard deviation) was $20.62 \pm 6.5$ in the control group and 23.29 \pm 5.8 in the case group. The Mann-Whitney's U-test showed no significant differences between the two groups regarding their pre-intervention MMSE score, suggesting that the two groups are comparable $(P=0.597)$. After the intervention, although the scores showed a considerable difference between the two groups, this difference was still not statistically significant $(P=0.109)$. However, the subtraction of the pre- and post-intervention scores was significantly different between the two groups, which suggests the positive effect of the administered medication $(P<0.0001$; Figures 1 and 2$)$.

Table 1. The mean and standard deviation and maximum and minimum age of the study subjects after eliminating the excluded patients

\begin{tabular}{llllccc}
\hline \multirow{4}{*}{ Male } & & Mean & N & SD & Min. & Max. \\
\hline \multirow{5}{*}{ Female } & Control & 62.00 & 19 & 13.287 & 31 & 86 \\
& Case & 59.92 & 13 & 17.937 & 27 & 78 \\
& Total & 61.16 & 32 & 15.104 & 27 & 86 \\
& Control & 66.73 & 15 & 15.281 & 30 & 85 \\
& Case & 57.10 & 21 & 14.666 & 28 & 84 \\
& Total & 61.11 & 36 & 15.477 & 28 & 85 \\
& Control & 64.09 & 34 & 14.179 & 30 & 86 \\
& Case & 58.18 & 34 & 15.789 & 27 & 84 \\
& Total & 61.13 & 68 & 15.188 & 27 & 86 \\
\hline
\end{tabular}

The Wilcoxon signed-rank test showed a significant difference between the pre-intervention and postintervention scores in each group $(P=0.024$ and $P<0.001$ respectively). However, the changes in scores were in opposite directions in the two groups; that is, the score reduced with time in the control group receiving placebos (as per the study protocol) but increased significantly in the case group receiving the medication.

\section{Discussion}

The present study was conducted in response to previous conflicting results on the subject. The findings showed an improved cognitive status in hemodialysis patients in the case (supplement) group compared to the control (placebo) group with the daily intake of CoQ10 100mg supplements $(P<0.001)$. Previous studies have also examined the effectiveness of CoQ10 supplements in preventing the progress of renal disease and have reported

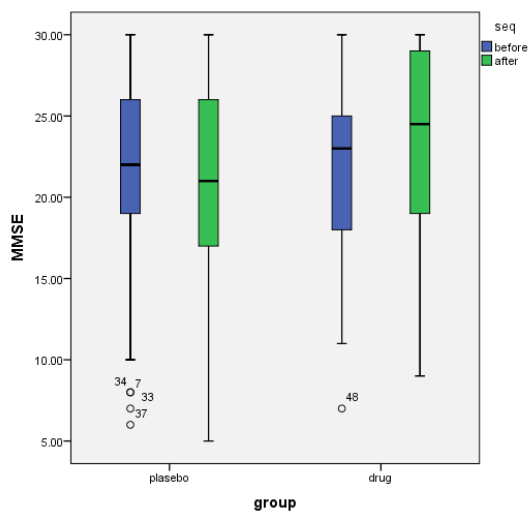

Figure 1. The box plot of the MMSE scores.

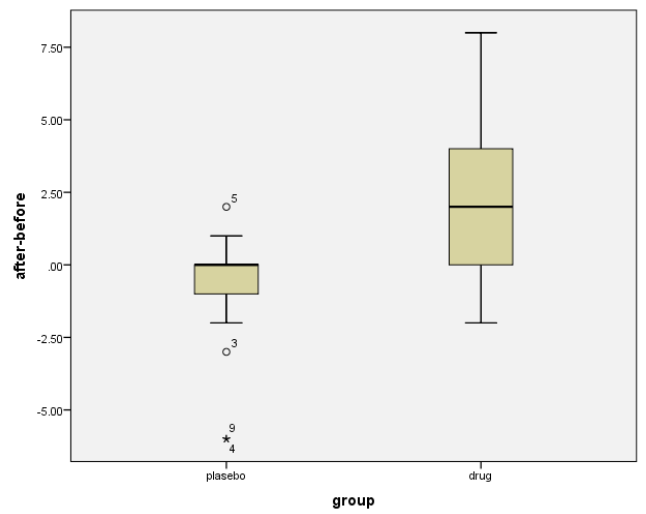

Figure 2. The box plot of the subtraction of the MMSE scores.

Table 2. The mean and standard deviation of the pre- and post-intervention MMSE scores in the two groups

\begin{tabular}{|c|c|c|c|c|c|c|c|c|c|}
\hline \multirow{2}{*}{ Group } & & \multirow{2}{*}{$\mathbf{N}$} & \multirow{2}{*}{ Mean } & \multirow{2}{*}{ SD } & \multirow{2}{*}{ Minimum } & \multirow{2}{*}{ Maximum } & \multicolumn{3}{|c|}{ Percentiles } \\
\hline & & & & & & & 25th & 50th (Median) & 75th \\
\hline \multirow{2}{*}{ Control } & MMSE1 & 34 & 21.29 & 6.562 & 7 & 30 & 18.50 & 22.00 & 26.25 \\
\hline & MMSE2 & 34 & 20.62 & 6.499 & 5 & 30 & 16.75 & 21.00 & 26.00 \\
\hline \multirow{2}{*}{ Case } & MMSE1 & 34 & 21.09 & 5.496 & 7 & 30 & 17.75 & 22.50 & 25.00 \\
\hline & MMSE2 & 34 & 23.29 & 5.818 & 9 & 30 & 18.75 & 24.50 & 29.00 \\
\hline
\end{tabular}


positive effects for this supplement. Several studies have also examined the cognitive status of hemodialysis patients and have found a greater relative severity of cognitive disorder in these patients. The effect of CoQ10 supplement on the cognitive status of hemodialysis patients has not been previously examined and the present study is the first attempt at the subject.

Bossola et al studied the cognitive status of chronic hemodialysis patients using the MMSE and assessed 80 hemodialysis patients and 160 older patients using the MMSE twice within an interval of one year. The patients in their study were divided into three groups based on their MMSE scores, including a normal cognitive function group, a mild to moderate cognitive disorder group and a severe cognitive disorder group. The mean one-year reduction in the MMSE score was higher in the hemodialysis group (from 24 to 21 ) than in the group of older patients (from 26 to 25$)(P=0.001)$. Compared to the older patients, a higher percentage of the hemodialysis patients progressed from a normal cognitive function to a mild to moderate and severe cognitive disorder $(P<1.0001)$. They concluded that a higher one-year reduction is observed in the MMSE score in the hemodialysis patients compared to the group of older patients and found no other factors that they could link to this higher reduction in the hemodialysis patients; however, reduced hemoglobin and cardiovascular morbidities are likely to affect this reduction (36).

Dahbour et al conducted a prospective study of MMSE before and after dialysis and compared 54 patients before their dialysis and two to four weeks after and compared them to their control group. Compared to the control group, the hemodialysis patients had clearly lower scores and hemodialysis thus had no effect on the scores obtained. This finding can be indicative of the poor sensitivity of this test in detecting cognitive disorder and distinguishing it from normal cognitive functioning (37).

In India, Thimmaiah et al studied the effect of renal failure on attention and memory before and 24 hours after hemodialysis. They gave the MMSE and the Brief Cognitive Rating Scale (BCRS) to 30 patients with renal failure and 30 healthy controls who were matching in terms of age, gender and socioeconomic status. Their findings revealed a higher cognitive status before dialysis in all the five different areas, i.e. attention, recent memory, past memory, orientation and self-care, compared to 24 hours after dialysis (at which point the subjects had the lowest cognitive status), which suggests a significant difference between the pre- and post-dialysis cognitive status $(P=0.001)$. Patients with renal failure can therefore substantially recover from cognitive disorder after the end of their hemodialysis sessions (38).

In the present study, the patients' mean pre-intervention MMSE score was 21.19. Other studies have also compared the patients' cognitive status with a control group using the MMSE; in this study, however, since the aim was to determine the effect of CoQ10 supplement on dialysis patients' cognitive status, the MMSE scores of the supplement group were not compared with a non-dialysis control group, rather, the changes in the cognitive status of hemodialysis patients under the effect of CoQ10 supplement were compared with a hemodialysis group that did not receive this supplement. Previous studies have also addressed the effect of CoQ10 supplementation in preventing the progress of renal disease and have reported positive effects for this supplement (39-42).

Gazdivoka et al studied 50 patients and determined their serum malondialdehyde (MDA) and select antioxidant levels (CoQ10, alpha-Tocopherol and beta-Carotene) in the conservative treatment of kidney patients and divided them into three groups based on their creatinine clearance. In their study, plasma concentrations of CoQ10 reduced with the supportive treatment of kidney patients and MDA levels increased despite the slight reduction in GFR. In the patients with advanced renal failure, betacarotene levels reduced. These changes affect the progress of kidney disease and their correction can thus prevent the progress of the disease (43).

Sakata et al studied the effect of the administration of CoQ10 on plasma oxidant levels and the antioxidant capacity in 36 hemodialysis patients over a six-month period. Advanced oxidation protein products (AOPP) and MDA and their percentage in the CoQ10 group were higher compared to the healthy controls before the administration of the coenzyme. Their study showed that the administration of CoQ10 has a relative suppressive effect on oxidative stress levels in hemodialysis patients (44).

Siaki et al studied serum CoQ10, MDA, super oxide dismutase (SOD) and antioxidant activity (AOA) levels in 40 CKD patients (in stages three to five of the disease) receiving supportive and non-dialysis treatments, 40 hemodialysis patients and 60 patients undergoing continuous ambulatory peritoneal dialysis (CAPD). They found no differences between the groups in terms of their serum CoQ10 levels; however, the antioxidant system (SOD and AOA) was substantially higher in the CAPD group compared to the CKD group. No differences were observed between the groups in their MDA levels either (45).

Rawn et al examined the effect of CoQ10 and antioxidant vitamin deficiency in the development of oxidative damage in patients with CRF in a double-blinded study in which they divided 21 dialysis and non-dialysis CRF patients (5 $\mathrm{mg} / \mathrm{dl}$ serum creatinine and higher) into an intervention $(\mathrm{n}=11)$ and a control group. Their intervention group was treated with 60-mg doses of CoQ10 three times per day and their control group was given $1 \mathrm{~g}$ of cellulose as a placebo three times per day for a total of four weeks. The results obtained showed a significant reduction in serum creatinine and urea levels and a significant increase in creatinine clearance and urinary output in the 
intervention group over the four weeks compared to the control group. They thus found that the administration of CoQ10 improves kidney function in patients with renal failure and reduces their need for dialysis. Nevertheless, long-term studies are required to confirm this finding (46).

Mehmetoglu et al studied the effect of oxidative stress on the balance between free radical products and their antioxidant activities in terms of the effect of CoQ10 on the normalization of the lipid level in 41 CAPD patients (21 men and 20 women), 38 hemodialysis patients (20 men and 18 women) and 43 healthy people ( 23 men and 20 women). Their results showed an increase in oxidative stress in the hemodialysis and CAPD groups compared to in the healthy group (47).

Ishii et al studied the relationship between oxidative stress biomarkers and clinical outcomes such as the overall rate of mortality, hospitalization and cardiovascular events in hemodialysis patients. The biomarkers tested included CoQ10 and biological antioxidant potential (BAP). They monitored 108 patients for a duration of 30 months and found no relationships between CoQ10 levels and clinical outcomes. They proposed a low BAP as an independent risk factor for mortality irrespective of a history of angiotensin inhibitor/receptor blocker intake (48).

\section{Conclusion}

The present study was conducted to determine the effectiveness of the intake of CoQ10 in improving cognitive status in chronic hemodialysis patients. The results obtained showed that the daily intake of $100 \mathrm{mg}$ of CoQ10 leads to a significant change in the cognitive status of chronic hemodialysis patients compared to when a placebo is administered. Since no similar studies have yet been conducted in this area, and given previous findings on the positive effect of CoQ10 supplementation in preventing the progress of kidney disease, the researchers recommend further studies to be conducted with larger sample sizes and with a healthy control group in order to establish a base measure for the healthy population and to thus enable the better interpretation of the effect of this supplement.

\section{Study limitations}

The study limitations include the failure to measure the patients' serum CoQ10 level before and after the intervention and the short supplementation period of only three months, which necessitate further studies with longer supplementation periods. Another limitation is the lack of a healthy control group for assessing the cognitive status of the general healthy population with the MMSE. The present study showed that the daily intake of 100 mg of CoQ10 for 12 weeks in conjunction with the usual medications can significantly improve the cognitive status of hemodialysis patients. Using CoQ10 as a supplement is therefore recommended.
Acknowledgments

Hereby, the authors would like to express their gratitude to the dialysis personnel of Kosar hospital and all those who helped us in conducting this study.

\section{Authors' contribution}

MRT was the principal investigators of the study. MRT and ShR participated in preparing the concept of design. MRT and MM revised the manuscript and critically evaluated the intellectual contents. All authors participated in preparing the final draft of the manuscript, revisited the manuscript, and critically evaluated the intellectual contents. All authors have read and approved the content of the manuscript and confirmed the accuracy or integrity of any part of the work.

\section{Conflicts of interest}

There were no points of conflicts to declare.

\section{Ethical considerations}

Ethical issues (including plagiarism, data fabrication, double publication) have been completely observed by the authors.

\section{Funding/Support}

This paper is extracted from the residential thesis of Shahab Rafaati, in the Department of Internal Medicine, Semnan University of Medical Sciences (Thesis \# 236D). The study was supported by Semnan University of Medical Sciences.

\section{References}

1. Coca SG, Singanamala S, Parikh CR. Chronic kidney disease after acute kidney injury: a systematic review and meta-analysis. Kidney Int. 2012;81:442-8. doi: 10.1038/ ki.2011.379.

2. Jha V, Garcia-Garcia G, Iseki K, Li Z, Naicker S, Plattner B, et al. Chronic kidney disease: global dimension and perspectives. Lancet. 2013;382:260-72. doi: 10.1016/S01406736(13)60687-X.

3. Qaseem A, Hopkins RH Jr, Sweet DE, Starkey M, Shekelle P. Screening, monitoring, and treatment of stage 1 to 3 chronic kidney disease: A clinical practice guideline from the American College of Physicians. Ann Intern Med. 2013;159:835-47.

4. Wetmore JB, Liu J, Li S, Hu Y, Peng Y, Gilbertson DT, et al. The healthy people 2020 objectives for kidney disease: how far have we come, and where do we need to go? Clin J Am Soc Nephrol. 2017;12:200-209. doi: 10.2215/CJN.04210416.

5. CDC. National chronic kidney disease fact sheet. Atlanta: Available from: https://www.cdc.gov/kidneydisease/pdf/ kidney_factsheet.pdf. 2014.

6. Xu W, Gerety P, Aleman T, Swanson J, Taylor J. Noninvasive methods of detecting increased intracranial pressure. Childs Nerv Syst. 2016;32:1371-86. doi: 10.1007/s00381016-3143-x.

7. Baumgaertel MW, Kraemer M, Berlit P. Neurologic complications of acute and chronic renal disease. Handb Clin Neurol. 2013;119:383-93. doi: 10.1016/B978-0-7020- 
4086-3.00024-2.

8. Chillon JM, Massy ZA, Stengel B. Neurological complications in chronic kidney disease patients. Nephrol Dial Transplant. 2016;31:1606-14. doi: 10.1093/ndt/gfv315.

9. Bugnicourt JM, Godefroy O, Chillon JM, Choukroun G, Massy ZA. Cognitive disorders and dementia in CKD: the neglected kidney-brain axis. J Am Soc Nephrol. 2013;24:353-63. doi: 10.1681/ASN.2012050536.

10. Drew DA, Weiner DE. Cognitive impairment in chronic kidney disease: keep vascular disease in mind. Kidney Int. 2014;85:505-7. doi: 10.1038/ki.2013.437.

11. Kurella Tamura M, Yaffe K, Hsu CY, Yang J, Sozio S, Fischer $\mathrm{M}$, et al. Cognitive impairment and progression of CKD. Am J Kidney Dis. 2016;68:77-83. doi: 10.1053/j. ajkd.2016.01.026.

12. Stringuetta-Belik F, Martin LC, Franco RJ. Cognitive impairment in chronic kidney disease. J Bras Nefrol. 2014;36:116-7.

13. da Matta SM, Janaina Matos M, Kummer AM, Barbosa IG, Teixeira AL, Silva AC. Cognitive alterations in chronic kidney disease: an update. J Bras Nefrol. 2014;36:241-5.

14. Darsie B, Shlipak MG, Sarnak MJ, Katz R, Fitzpatrick AL, Odden MC. Kidney function and cognitive health in older adults: the Cardiovascular Health Study. Am J Epidemiol. 2014;180:68-75. doi: 10.1093/aje/kwu102.

15. Wang H, Fang C, Cai L, Dong B, Deng J. Chronic kidney disease and cognitive impairment among the very old in China. Aging Clin Exp Res. 2016;28:475-82. doi: 10.1007/ s40520-015-0433-1.

16. Kallenberg MH, Kleinveld HA, Dekker FW, van Munster BC, Rabelink TJ, van Buren $M$, et al. Functional and cognitive impairment, frailty, and adverse health outcomes in older patients reaching ESRD-a systematic review. Clin J Am Soc Nephrol. 2016;11:1624-39. doi: 10.2215/ CJN.13611215.

17. O’Lone E, Connors M, Masson P, Wu S, Kelly PJ, Gillespie $\mathrm{D}$, et al. Cognition in people with end-stage kidney disease treated with hemodialysis: a systematic review and metaanalysis. Am J Kidney Dis. 2016;67:925-35. doi: 10.1053/j. ajkd.2015.12.028.

18. Shea YF, Lam MF, Lee MS, Mok MY, Lui SL, Yip TP, et al. Prevalence of cognitive impairment among peritoneal dialysis patients, impact on peritonitis and role of assisted dialysis. Perit Dial Int. 2016;36:284-90. doi: 10.3747/ pdi.2014.00247.

19. Liu WC, Zheng CM, Lu CL, Lin YF, Shyu JF, Wu CC, et al. Vitamin D and immune function in chronic kidney disease. Clin Chim Acta. 2015;450:135-44. doi: 10.1016/j. cca.2015.08.011.

20. Meijers RW, Betjes MG, Baan CC, Litjens NH. T-cell ageing in end-stage renal disease patients: Assessment and clinical relevance. World J Nephrol. 2014;3:268-76. doi: 10.5527/ wjn.v3.i4.268.

21. Scholze A, Jankowski J, Pedraza-Chaverri J, Evenepoel P. Oxidative stress in chronic kidney disease. Oxid Med Cell Longev. 2016;2016:8375186. doi: 10.1155/2016/8375186.

22. Sung SH, Jang IS. Isolated central nervous system relapse of acute lymphoblastic leukemia. Brain Tumor Res Treat. 2014;2:114-8. doi: 10.14791/btrt.2014.2.2.114.

23. Kennedy DJ, Tang WH, Fan Y, Wu Y, Mann S, Pepoy M, et al. Diminished antioxidant activity of high-density lipoprotein-associated proteins in chronic kidney disease. J Am Heart Assoc. 2013;2:e000104. doi: 10.1161/
JAHA.112.000104.

24. Alam S, Siddiqui MR. Accelerated atherosclerosis in patients with Chronic Kidney Disease - the role of traditional and non-traditional risk factors. WebmedCentral MEDICINE 2014;5:WMC004759. doi: 10.9754/journal. wmc.2014.004759.

25. Shah SV, Shukla AM, Bose C, Basnakian AG, Rajapurkar M. Recent advances in understanding the pathogenesis of atherosclerosis in CKD patients. J Ren Nutr. 2015;25:205-8. doi: 10.1053/j.jrn.2014.10.024.

26. Lee BJ, Tseng YF, Yen CH, Lin PT. Effects of coenzyme Q10 supplementation (300 mg/day) on antioxidation and antiinflammation in coronary artery disease patients during statins therapy: a randomized, placebo-controlled trial. Nutr J. 2013;12:142. doi: 10.1186/1475-2891-12-142.

27. Claessens AJ, Yeung CK, Risler LJ, Phillips BP, Himmelfarb J, Shen DD. Rapid and sensitive analysis of reduced and oxidized coenzyme Q10 in human plasma by UPLCMS/MS and application to studies in healthy human subjects. Ann Clin Biochem. 2016;53:265-73. doi: 10.1177/0004563215593097.

28. Small DM, Gobe GC. Oxidative stress and antioxidant therapy in chronic kidney and cardiovascular disease. In: Morales-Gonzalez JA, ed. Oxidative stress and chronic degenerative diseases-a role for antioxidants/Rijeka, Croatia: InTech. 2013. p. 233-64.

29. Macunluoglu B, Kaya Y, Atakan A, Ari E, Kaspar C, Demir $\mathrm{H}$, et al. Serum coenzyme Q10 levels are associated with coronary flow reserve in hemodialysis patients. Hemodial Int. 2013;17:339-45. doi: 10.1111/hdi.12001.

30. Tian G, Sawashita J, Kubo H, Nishio SY, Hashimoto S, Suzuki N, et al. Ubiquinol-10 supplementation activates mitochondria functions to decelerate senescence in senescence-accelerated mice. Antioxid Redox Signal. 2014;20:2606-20. doi: 10.1089/ars.2013.5406.

31. Labban L. The Benefits of Coenzyme Q10 as A Nutritional and Medicinal Supplement. EC Nutrition. 2015;2:302-11.

32. Fukuda S, Nojima J, Kajimoto O, Yamaguti K, Nakatomi Y, Kuratsune $\mathrm{H}$, et al. Ubiquinol-10 supplementation improves autonomic nervous function and cognitive function in chronic fatigue syndrome. Biofactors. 2016;42:431-40. doi: 10.1002/biof.1293.

33. Mantle D. The pharmacology of coenzyme Q 10: its relevance to health. Br J Card Nurs. 2013;8:525-530.

34. Niedzielska E, Smaga I, Gawlik M, Moniczewski A, Stankowicz P, Pera J, et al. Oxidative stress in neurodegenerative diseases. Mol Neurobiol. 2016;53:40944125 .

35. Seyedian M, Falah M, Nourouzian M, Nejat S, Delavar A, Ghasemzadeh H. Validity of the Farsi version of minimental state examination. J Med Council IRI. 2008;25:408414.

36. Bossola M, Antocicco M, Di Stasio E, Ciciarelli C, Luciani G, Tazza L, et al. Mini Mental State Examination over time in chronic hemodialysis patients. J Psychosom Res. 2011;71:50-4. doi: 10.1016/j.jpsychores.2011.01.001.

37. Dahbour SS, Wahbeh AM, Hamdan MZ. Mini mental status examination (MMSE) in stable chronic renal failure patients on hemodialysis: The effects of hemodialysis on the MMSE score. A prospective study. Hemodial Int. 2009;13:80-5. doi: 10.1111/j.1542-4758.2009.00343.x.

38. Thimmaiah R, Murthy KK, Pinto D. Cognitive dysfunction in patients with renal failure requiring hemodialysis. 
Indian J Psychol Med. 2012;34:237-41. doi: 10.4103/02537176.106019 .

39. Zahed NS, Ghassami M, Nikbakht H. Effects of coenzyme Q10 supplementation on C-reactive protein and homocysteine as the inflammatory markers in hemodialysis patients; a randomized clinical trial. J Nephropathol. 2016;5:38-43. doi: 10.15171/jnp.2016.07.

40. Yeung CK, Billings FT, Claessens AJ, Roshanravan B, Linke L, Sundell MB, et al. Coenzyme Q 10 dose-escalation study in hemodialysis patients: safety, tolerability, and effect on oxidative stress. BMC Nephrol. 2015;16:183. doi: 10.1186/ s12882-015-0178-2.

41. Nasri H. Antioxidant therapy for hemodialysis patients. Ann Res Antioxid. 2016;1:e01.

42. Tamay-Cach F, Quintana-Pérez JC, Trujillo-Ferrara JG, Cuevas-Hernández RI, Del Valle-Mondragón L, GarcíaTrejo EM, et al. A review of the impact of oxidative stress and some antioxidant therapies on renal damage. Ren Fail. 2016;38:171-5. doi: 10.3109/0886022X.2015.1120097.

43. Gazdikova K, Gvozdjakova A, Kucharska J, Spustova V, Braunova Z, Dzurik R. Malondialdehyde and selected antioxidants in conservatively treated patients with kidney diseases. Bratisl Lek Listy. 2000;101:490-4.
44. Sakata T, Furuya R, Shimazu T, Odamaki M, Ohkawa S, Kumagai H. Coenzyme Q10 administration suppresses both oxidative and antioxidative markers in hemodialysis patients. Blood Purif. 2008;26:371-8. doi: 10.1159/000135605.

45. Saiki R, Lunceford AL, Shi Y, Marbois B, King R, Pachuski J, et al. Coenzyme Q10 supplementation rescues renal disease in Pdss $2 \mathrm{kd} / \mathrm{kd}$ mice with mutations in prenyl diphosphate synthase subunit 2. Am J Physiol Renal Physiol. 2008;295:F1535-44. doi: 10.1152/ajprenal.90445.2008.

46. Singh RB, Khanna HK, Niaz MA. Randomized, doubleblind placebo-controlled trial of coenzyme Q10 in chronic renal failure: discovery of a new role. J Nutr Environ Med. 2000;10:281-8

47. Mehmetoglu I, Yerlikaya FH, Kurban S, Erdem SS, Tonbul Z. Oxidative stress markers in hemodialysis and peritoneal dialysis patients, including coenzyme Q10 and ischemiamodified albumin. Int J Artif Organs. 2012;35:226-32. doi: 10.5301/ijao.5000078.

48. Ishii $\mathrm{T}$, Ohtake $\mathrm{T}$, Okamoto $\mathrm{K}$, Mochida $\mathrm{Y}$, Ishioka $\mathrm{K}$, Oka $\mathrm{M}$, et al. Serum biological antioxidant potential predicts the prognosis of hemodialysis patients. Nephron Clin Pract. 2011;117:c230-6. doi: 10.1159/000320201.

Copyright $\odot 2018$ The Author(s); Published by Society of Diabetic Nephropathy Prevention. This is an open-access article distributed under the terms of the Creative Commons Attribution License (http://creativecommons.org/licenses/by/4.0), which permits unrestricted use, distribution, and reproduction in any medium, provided the original work is properly cited. 